\title{
A JET MODEL FOR THE BROADBAND SPECTRUM OF THE SEYFERT 1 GALAXY NGC 4051
}

\author{
Dipankar Maitra ${ }^{1}$, Jon M. Miller $^{1}$, Sera MarkofF${ }^{2}$, and Ashley King ${ }^{1}$ \\ ${ }^{1}$ Department of Astronomy, University of Michigan, Ann Arbor, MI 48109, USA; dmaitra@umich.edu \\ 2 Astronomical Institute “Anton Pannekoek,” University of Amsterdam, P.O. Box 94249, 1090 GE Amsterdam, The Netherlands \\ Received 2011 January 26; accepted 2011 April 26; published 2011 June 22
}

\begin{abstract}
Recent radio very long baseline interferometry observations of the $\sim$ parsec-scale nuclear region of the narrow line Seyfert 1 galaxy NGC 4051 hint toward the presence of outflowing plasma. From available literature we have collected high-quality, high-resolution broadband spectral energy distribution (SED) data of the nuclear region of NGC 4051 spanning from radio through X-rays, to test whether the broadband SED can be explained within the framework of a relativistically outflowing jet model. We show that once the contribution from the host galaxy is taken into account, the broadband emission from the active galactic nucleus (AGN) of NGC 4051 can be well described by the jet model. Contributions from dust and ongoing star formation in the nuclear region tend to dominate the IR emission even at the highest resolutions. In the framework of the jet model, the correlated high variability of the extreme-ultraviolet and X-rays compared to other wavelengths suggests that the emission at these wavelengths is optically thin synchrotron originating in the particle acceleration site(s) in the jet very close (few $r_{\mathrm{g}}=G M_{\mathrm{BH}} / c^{2}$ ) to the central supermassive black hole of mass $M_{\mathrm{BH}}$. Our conclusions support the hypothesis that narrow line Seyfert 1 galaxies (which NGC 4051 is a member of) harbor a "jetted" outflow with properties similar to what has already been seen in low-luminosity AGNs and stellar mass black holes in hard X-ray state.
\end{abstract}

Key words: galaxies: active - galaxies: individual (NGC 4051) - galaxies: nuclei - galaxies: Seyfert

Online-only material: color figures

\section{INTRODUCTION}

While it is generally agreed that the enormous energy output of active galactic nuclei (AGNs) as well as X-ray binaries (XRBs) is due to accretion of matter onto a compact object $\left(G M /\left[c^{2} R\right] \sim 1\right.$; see, e.g., Rees 1984 for a review), the fate of the accreted material is somewhat uncertain. Most likely, a large fraction of this accreted matter crosses the event horizon of the black hole and is forever lost from the observer's view. However, the presence of relativistic outflows, often dubbed "jets," observed to emanate from the very core of the AGNs and XRBs, shows that some of the accreted material is in fact ejected from the inner accretion flow. Recent simulations show that such jets are likely Poynting flux dominated, i.e., only a very small fraction of the accreted material is ejected while most of the energy in the jets is carried by the electromagnetic fields. Launching of such relativistic outflows, their composition, and their association with the accretion inflow are currently topics of intense research. Observations indicate that nuclear radio loudness of AGNs (parameterized by the radio-to-optical luminosity ratio) tends to increase with decreasing Eddingtonscaled nuclear luminosity (Ho 2002; Sikora et al. 2007). A similar situation is seen in XRBs accreting at low mass accretion rates where the radio loudness (in this case parameterized by radio-to-soft X-ray flux ratio) tends to increase with decreasing X-ray luminosity (Fender et al. 2003). The radio emission from a radio-loud AGN is generally attributed to synchrotron emission from a population of nonthermal particles in the jets and the lobes. But the emission mechanism in radio-quiet AGNs such as Seyferts remains much less well understood. Recent very long baseline interferometry (VLBI) observations reported by Giroletti \& Panessa (2009) have detected radio emission from the nuclear region of four very faint Seyfert galaxies (NGC 4051, NGC 4388, NGC 4501, and NGC 5033), where high brightness temperature $\left(T_{\mathrm{B}} \sim 10^{5}-10^{7} \mathrm{~K}\right)$ and (in some cases) resolved structure in the core favor a weak jet/outflow origin for these sources.

Among these four faint Seyferts studied by Giroletti \& Panessa (2009), NGC 4051, one of the "original" Seyfert galaxies studied by Seyfert (1943) due to its strong nuclear emission lines, is a narrow line Seyfert 1 galaxy (NLS1; see Osterbrock \& Pogge 1985 for definition of NLS1s) that has been well studied in almost all wavelengths from radio to the X-rays. Located in the Ursa Major cluster of galaxies, NGC 4051 is a fairly nearby $(z=0.002336 \pm 0.000004)$ spiral galaxy. Based on the latest reverberation mapping estimates, the AGN in the heart of NGC 4051 boasts a supermassive black hole (SMBH) of $(1.7 \pm 0.5) \times 10^{6} M_{\odot}$ (Denney et al. 2009). As mentioned above, recent VLBI observations of NGC 4051 at 1.6 and $5 \mathrm{GHz}$ show a complex, sub-parsec-scale compact core and other structure (Giroletti \& Panessa 2009). Similar results have also been obtained from recent Very Large Array (VLA)/Expanded Very Large Array (EVLA) observations (King et al. 2011; Jones et al. 2011). The high brightness temperature of the compact core of NGC $4051\left(>1.8 \times 10^{5} \mathrm{~K}\right)$ along with the observed radio structures leads to the conclusion that the radio emission may be nonthermal in origin and likely originating from an outflow/jet.

From Gemini Near-infrared Integral Field Spectrograph (NIFS) data of NGC 4051, Riffel et al. (2008) have found a strong $\mathrm{H}$ II $\mathrm{Br} \gamma$ emission at $2.1661 \mu \mathrm{m}$ in the nuclear spectrum (see their Figure 1). Based on the $\mathrm{H}_{2} \lambda 2.1218 \mu \mathrm{m} / \mathrm{Br} \gamma$ line ratio they concluded that some contribution to the strong $\mathrm{Br} \gamma$ emission is from shocks or energy injection due to interaction of the $\mathrm{H}_{2}$ emitting gas with the jet. While the strong $\mathrm{Br} \gamma$ line may be associated with interaction with the jet, it is quite unlikely that the IR continuum emission is dominated by the jet. Earlier high time resolution monitoring at $2.2 \mu \mathrm{m}$ continuum emission of NGC 4051 by Hunt et al. (1992) does not show any rapid IR variability (relative to X-rays) which might be expected if 
the dominant fraction of the observed IR emission is an extension of the optically thin X-ray emission (e.g., as recently found for the stellar black hole binary system XTE J1550-564 by Russell et al. 2010). Simultaneous optical, IR, and X-ray observations of NGC 4051 obtained by Done et al. (1990) also show little optical/IR variability compared to the X-rays, suggesting different origins for the X-ray continuum and optical/IR continuum emission.

Compact, steady jets are observed from XRBs when they are in hard X-ray state, i.e., their X-ray spectrum is dominated by hard power-law emission and large rms variability. In contrast, such steady, compact jets are not seen when XRBs are in the soft X-ray state characterized by a predominantly thermal spectrum from the accretion disk and low rms variability. See, e.g., Homan \& Belloni (2005) and Remillard \& McClintock (2006) for extensive definitions and review of X-ray states in XRBs, and Fender (2006) for a review of XRB jets. It is still not clear which physical mechanism(s) trigger transition between these states, as well as the associated change in disk-jet coupling. Such state transitions and appearance/disappearance of radio jets are now regularly seen in transient XRB systems (where epochs of activity typically span $\sim$ month or less), thanks to advances in instrumentation and coordinated, quasi-simultaneous multiwavelength observations over the past decade. Such changes however cannot be seen in AGN because the associated timescales can easily exceed the human lifespan, and advances can only be made via studying ensemble properties of AGN. Indeed, recent works show that there exists a tight relationship connecting the radio luminosity, X-ray luminosity, and black hole mass. This relationship, often referred to as the "fundamental plane" of black hole accretion (Merloni et al. 2003; Falcke et al. 2004), not only holds across the stellar mass black holes in hard state and low-luminosity AGNs (LLAGN), but also for SMBHs with direct, dynamical mass measurements (i.e., the $M-\sigma$ AGNs; see Gültekin et al. 2009), and extends to blazars as well (R. Plotkin et al. 2011, in preparation).

McHardy et al. (2004) have argued that NGC 4051 and the stellar mass black hole Cygnus X-1 in soft state have very similar Fourier power density spectra, and therefore NGC 4051 may be a soft state AGN accreting at $\sim 30 \% L_{\text {Edd }}$. Based on the bending frequency of the Fourier power spectrum of NGC 4051, these authors had deduced a central black hole mass of $3_{-1}^{+2} \times 10^{5} M_{\odot}$, which was consistent with the best estimated reverberation mass of $5_{-3}^{+6} \times 10^{5} M_{\odot}$ at that time. However, the most recent, revised reverberation mass estimate of $1.73_{-0.52}^{+0.55} \times 10^{6} M_{\odot}$ by Denney et al. (2009) is $\sim 5.7$ times larger, consequently lowering the bolometric luminosity to $\sim 5 \% L_{\text {Edd }}$. Moreover, as discussed in detail in their discussion section, the assumed X-ray to bolometric conversion factor of 27 is quite likely an overestimate. On the other hand, another commonly used empirical relationship to estimate the bolometric luminosity of similar AGNs is $L_{\text {bol }} \approx 9 \lambda L_{\lambda}$ (5100 ̊) (Kaspi et al. 2000; Peterson et al. 2004). Given NGC 4051's host galaxy starlight subtracted average nuclear flux density at $5100 \AA$ is $(4.5 \pm 0.4) \times 10^{-15} \mathrm{erg} \mathrm{s}^{-1} \mathrm{~cm}^{-2} \AA^{-1}$ (Denney et al. 2009), its bolometric luminosity is roughly $\approx(1 \%)(D / 10 \mathrm{Mpc})^{2}\left(M_{\mathrm{BH}} / 1.7 \times 10^{6} M_{\odot}\right)^{-1} L_{\mathrm{Edd}}$. Both of the above estimates of the bolometric luminosity are closer to Galactic black hole binaries in hard state than in soft state. It is also worth noting that Cygnus X-1 is actually never in a "classical" soft state, but rather it is always somewhat transitional (see, e.g., Nowak et al. 2005; Wilms et al. 2006). Thus, it is unclear whether NGC 4051 is indeed a soft state
AGN, or in hard state, or perhaps in a transitory state between canonical hard and soft states. Can XRB state definitions be straightforwardly carried over to AGNs? Such are the questions which motivate the current work.

Since jets are known to emit over a broad range of the electromagnetic spectrum spanning from radio through X-rays, it is plausible that at least some of the observed nuclear emission from NGC 4051 might also originate in the jet. In this work we have collected high-quality, high-resolution nuclear spectral energy distributions (SEDs) of NGC 4051 from the literature, to study the broadband (radio-X-ray) SED and test whether the observed nuclear emission can originate in a mildly relativistic jet outflow. We use the accretion disk+jet model developed by Markoff et al. (2005) and Maitra et al. (2009a) to model the broadband continuum. The model has been successfully applied to explaining broadband SEDs of several black hole XRBs in hard state, as well as those of the LLAGNs Sagittarius A* and M81* (see, e.g., Markoff 2010, and references therein). These previous results suggest that despite a difference of $\sim 5-6$ orders of magnitude in mass, the accretion/ejection processes in stellar mass black holes $\left(\sim 10 M_{\odot}\right)$ and SMBHs in the nearby LLAGNs $\left(\sim 10^{6-7} M_{\odot}\right)$ appear quite similar when comparing sources with similar Eddington-scaled luminosity.

This is the first time we apply this jet model to a radio-quiet Seyfert galaxy to test whether the broadband emission originates in a collimated jet outflow. The sources from which we obtained the multiwavelength SED data are described in Section 2, and a brief summary of the model parameters that best match the data is given in Section 3. Implications of the modeling results and conclusions drawn from the study are discussed in Section 4.

\section{THE BROADBAND SED OF NGC 4051}

The nuclear region of NGC 4051 has been observed with many different ground-based as well as space-borne telescopes spanning from radio through $\mathrm{X}$-rays, at the highest spatial resolution available in each of these wavelength ranges. The high angular resolution is of crucial importance for isolating the central, nuclear emission from that of the surrounding galaxy. This is especially important in optical and IR wavelengths where contributions from non-AGN components such as the host galaxy's stellar population, ongoing star formation near the nuclear region, and dust can easily dominate the observed emission if the photometric aperture is not small.

\subsection{X-ray}

The X-ray flux from NGC 4051 is known to be highly variable on a timescale of days (see, e.g., McHardy et al. 2004; Ponti et al. 2006; Breedt et al. 2010; Vaughan et al. 2011). Like most NLS1s, the 2-10 keV X-ray continuum SED of NGC 4051 is well modeled by a power law. A narrow iron line is seen near $6.4 \mathrm{keV}$, and the presence of curvature at higher energies hints toward the presence of reflection (Ponti et al. 2006; Miller et al. 2010). Similar to the X-ray spectra of many other NLSs, there exists an excess of soft photons in addition to the power law at $<2 \mathrm{keV}$ (see, e.g., Boller et al. 1996, also see Section 4). In this work we are interested in the power-law continuum only and take the power-law photon index $(\Gamma)$ and normalization from a recent simultaneous radio (VLA/EVLA)-X-ray (Chandra) campaign of NGC 4051 published by King et al. (2011). We chose this set of X-ray observations because they were performed simultaneously with the radio observations at $8.4 \mathrm{GHz}$. The solid black lines in Figure 1 show the power-law continuum measured from this Chandra data set. As discussed in 


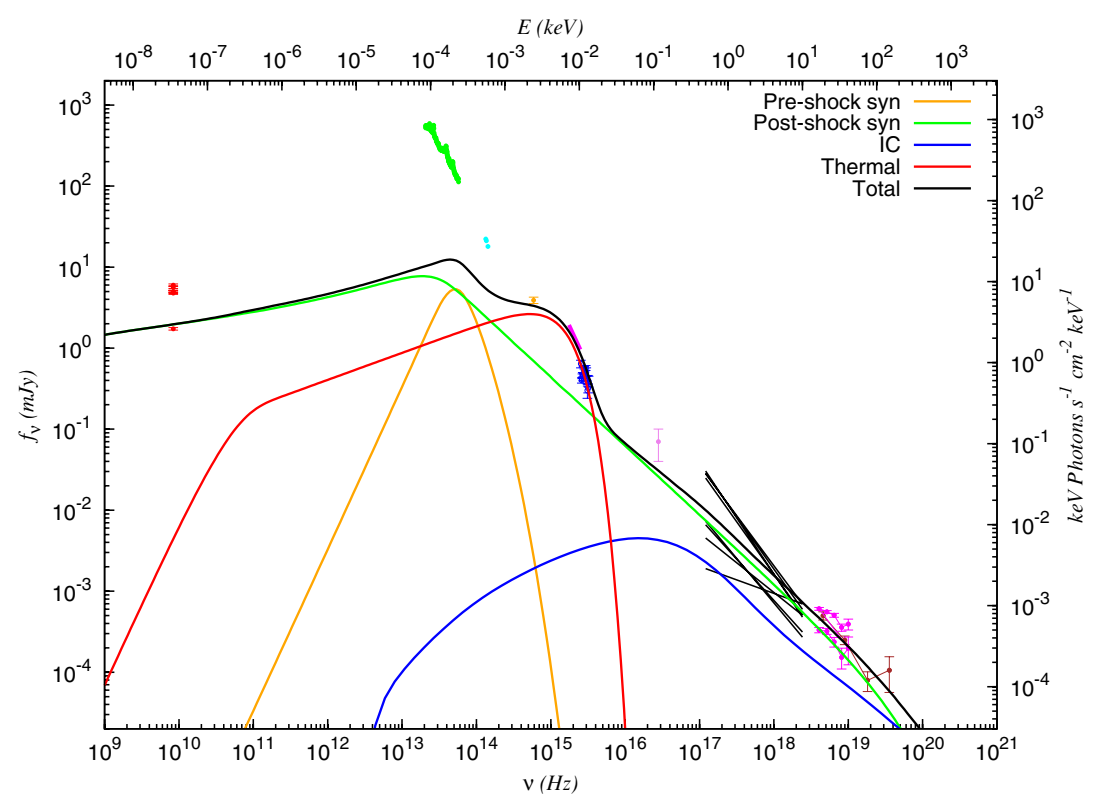

Figure 1. Broadband SED of NGC 4051. Chandra/ACIS (solid black lines) and VLA 8.4 GHz (red points) data taken from King et al. (2011). Suzaku/PIN data (magenta points connected by lines) from Miller et al. (2010). Swift/BAT data (brown points connected by line) from publicly available NASA archives. EUVE data (violet) from Uttley et al. (2000), FUSE data (blue points) are from Kaspi et al. (2004), HST/STIS fluxes (magenta line) from Collinge et al. (2001), and 5100 continuum data point (orange) from Denney et al. (2009). The Gemini/NIFS data (Riffel et al. 2008) and the Spitzer/IRS data (Sani et al. 2010) are shown in cyan and green, respectively. The SED was corrected for Galactic absorption assuming a column density of $E(B-V)=0.013$ and $N_{\mathrm{H}}=1.3 \times 10^{20} \mathrm{~cm}^{-2}$ in the direction of NGC 4051 (Elvis et al. 1989), and assuming the extinction law of Cardelli et al. (1989). The fits to high-resolution Chandra spectra discussed in King et al. (2011) suggest that intrinsic absorption is negligible. The thick solid lines show the various model components as well as the total model predicted SED.

(A color version of this figure is available in the online journal.)

Section 4 , the hard X-ray $(>10 \mathrm{keV})$ variability may be smaller than the soft $(2-10 \mathrm{keV}) \mathrm{X}$-ray variability. Therefore, we have also added two Suzaku/PIN spectra of NGC 4051 from the data presented by Miller et al. (2010). While NGC 4051 has not been detected in the Fermi/LAT data so far $(0.1-100 \mathrm{GeV}$; Abdo et al. 2010), it has been detected in the time averaged Swift/BAT (14-195 keV) data. The BAT spectrum ${ }^{3}$ is also included in Figure 1. Fits to the high-resolution Chandra HETGS spectra of NGC 4051 (A. King et al. 2011, in preparation) suggest that any absorption intrinsic to the source is negligible, therefore to correct for absorption we use a column density of $N_{\mathrm{H}}=1.3 \times 10^{20} \mathrm{~cm}^{-2}$ in the direction of NGC 4051 (Elvis et al. 1989).

\section{2. $U V$}

The Extreme Ultraviolet Explorer (EUVE) data are from Uttley et al. (2000), and the corresponding error bar represents the EUV variability range (also see Figure 7 of Kaspi et al. 2004). Uttley et al. (2000) noted that the EUV variability is comparable to the X-ray variability, and that the EUV and $\mathrm{X}$-rays were simultaneous to within $1 \mathrm{ks}$, which suggests a common origin for both EUV and soft X-ray emission.

The far-ultraviolet data in the wavelength range of 900-1180 ̊ are taken from the Far Ultraviolet Spectroscopic Explorer (FUSE) observations presented by Kaspi et al. (2004). Even though the three FUSE observations were separated by as much as $\sim 1$ year, the analysis by Kaspi et al. (2004) suggests little or no $(<10 \%)$ variation in the FUSE spectra. As discussed above, we are interested in the continuum emission only, and therefore use the average flux-density measurements

\footnotetext{
3 Obtained from http://heasarc.gsfc.nasa.gov/docs/swift/results/bs9mon/ bs_ind.php?number $=85$.
}

in wavelength ranges that are free from emission and absorption as presented in Table 2 of Kaspi et al. (2004).

We have also included Hubble Space Telescope (HST)/Space Telescope Imaging Spectrograph (STIS) data of NGC 4051 published by Collinge et al. (2001). The binned STIS spectrum presented in their work shows that the continuum flux is roughly constant at $\sim 2 \times 10^{-14}$ erg cm $\mathrm{cm}^{-2} \mathrm{~s}^{-1} \AA^{-1}$ over the observed wavelength range of $1200-1700 \AA$. These HST observations were made simultaneously with Chandra, and even though the Chandra light curve showed large variations in the X-ray flux (see, e.g., Figure 2 of Collinge et al. 2001), no variability could be detected in the STIS data. The lack of UV variability strongly contrasts with the high X-ray variability and suggests that the $\mathrm{UV}$ and X-rays are most likely coming from different emission mechanisms.

\subsection{Optical}

Contribution of non-AGN components, e.g., light from stars as well as dust, starts becoming important in optical and IR, and extreme caution must be taken not only to use the highest resolution available to observe the nuclear region, but also to model and subtract the non-AGN contributions within the aperture. Readily available optical and IR fluxes listed, e.g., in the NED, are therefore not usable for this work since the apertures listed in NED are typically large and there is no easy way to estimate the non-AGN contribution. Instead we use the results obtained by Denney et al. (2009), who presented new, optical spectroscopic and photometric observations of NGC 4051 from a densely sampled reverberation mapping campaign, using data from five different observatories $(1.3 \mathrm{~m}$ McGraw-Hill telescope at MDM Observatory, 0.7 and $2.6 \mathrm{~m}$ telescopes of the Crimean Astrophysical Observatory ( $\mathrm{CrAO}$ ), $2 \mathrm{~m}$ MAGNUM telescope at the Haleakala Observatories, and 
Table 1

Observations

\begin{tabular}{|c|c|c|c|}
\hline Wavelength or Energy Range & Observatory/Instrument & Observation Date(s) & Reference \\
\hline $3.5 \mathrm{~cm}$ & VLA/EVLA & 2008 Dec 31-2009 Jul 31 & King et al. (2011) \\
\hline $5-14 \mu \mathrm{m}$ & Spitzer/IRS & 2005 Dec 11 & Sani et al. (2010) \\
\hline $2-2.4 \mu \mathrm{m}$ & Gemini/NIFS & 2006 Jan & Riffel et al. (2008) \\
\hline $5100 \AA$ & $\begin{array}{l}\mathrm{MDM} / 1.3 \mathrm{~m}, \mathrm{MAGNUM} / 2 \mathrm{~m}, \\
\mathrm{CrAO} /(0.7 \mathrm{~m} \text { and } 2.6 \mathrm{~m}) \\
\mathrm{UNebr} / 0.4 \mathrm{~m}\end{array}$ & 2007 Mar 20-2007 Jul 30 & Denney et al. (2009) \\
\hline $1173-1730 \AA$ & $H S T / \mathrm{STIS}$ & 2000 Mar 24-25 & Collinge et al. (2001) \\
\hline $900-1180 \AA$ & FUSE & 2002 Mar 29, 2003 Jan 18, Mar 19 & Kaspi et al. (2004) \\
\hline $70-190 \AA$ & $E U V E$ & 1996 May 20-28, Dec 11-14 & Uttley et al. (2000) \\
\hline $0.5-10 \mathrm{keV}$ & Chandra/ACIS & 2009 Jan 7 - 2009 Jul 31 & King et al. (2011) \\
\hline $15-60 \mathrm{keV}$ & Suzaku/PIN & 2005 Nov $10-13,2008$ Nov 6-12 & Miller et al. (2010) \\
\hline $15-200 \mathrm{keV}$ & Swift/BAT & Time averaged since start of mission & Publicly available \\
\hline
\end{tabular}

the $0.4 \mathrm{~m}$ telescope of the University of Nebraska (UNebr)). They then subtract the host galaxy starlight contribution to the continuum flux following the prescription by Bentz et al. (2009). The estimated AGN continuum flux at $5100 \AA$ for NGC 4051 is $(4.5 \pm 0.4) \times 10^{-15} \mathrm{erg} \mathrm{s}^{-1} \mathrm{~cm}^{-2} \AA^{-1}$ after subtracting the host galaxy starlight contribution. The host galaxy starlight contribution is about twice that of the AGN. However, the optical flux is much less variable than the X-ray and EUV. The excess variance in the $5100 \AA$ measurements of Denney et al. (2009) is 0.09 . A recent analysis of the last 12 years of X-ray and optical variability in NGC 4051 by Breedt et al. (2010) also concluded that the variability of the AGN in optical wavelengths (where the fractional variability, $F_{\mathrm{var}}$, is $\sim 12 \%-26 \%$ ) is much smaller than the variability in X-rays $\left(F_{\mathrm{var}} \sim 49 \%\right)$.

\subsection{Infrared}

For IR we used the Spitzer mid-infrared (MIR; 5-15 $\mu \mathrm{m}$ ) spectrum of NGC 4051 published by Sani et al. (2010) and also adaptive optics Integral Field Unit spectroscopic data reported by Riffel et al. (2008) which were obtained with the NIFS mounted on the Gemini North telescope. Three representative continuum fluxes at $2.1,2.2$, and $2.25 \mu \mathrm{m}$ from the nuclear (central $0^{\prime \prime} .1 \times 00^{\prime \prime} 1$ ) region obtained by the NIFS are shown by the cyan points in Figure 1.

The Spitzer spectrum (also see Figure 2 of Sani et al. 2010) shows strong polycyclic aromatic hydrocarbon (PAH) features due to ongoing star formation activity. According to Sani et al. (2010), the MIR continuum may also be primarily due to thermal emission by AGN-heated dust. Therefore, the contribution of direct AGN emission in MIR appears to be small. The slit width of the IR spectrograph is 3.6 arcsec, which at the distance of NGC 4051 implies a linear scale of $\sim 175(D / 10 \mathrm{Mpc}) \mathrm{pc}$. The Spitzer/Infrared Spectrograph (IRS) slit width is a factor of 18 larger than that of the HST/STIS slit width $(0.2$ arcsec $)$ and the former therefore includes significantly larger circumnuclear regions. The Spitzer data therefore do not put strong constraints on our broadband modeling. However, they put upper limits on the direct (accretion/ejection origin) AGN emission in the MIR wavelengths. The Gemini NIFS nuclear spectrum with an angular coverage of $0^{\prime \prime} 1 \times 00^{\prime \prime} 1$ is expected to contain a significantly larger fraction of AGN-to-non-AGN (starburst, dust) emission compared to the IRS.

\subsection{Radio}

The $8.4 \mathrm{GHz}$ radio data are taken from King et al. (2011), where the authors present simultaneous radio and X-ray monitoring observations of NGC 4051. During this campaign NGC 4051 was observed six times between 2008 December 31 and 2009 July 31 simultaneously using both VLA/EVLA GHz and Chandra. Details of the radio data analysis are presented in King et al. (2011).

The UV, optical, and IR data were dereddened assuming $E(B-V)=0.013 \mathrm{mag}$ and using the extinction law of Cardelli et al. (1989). We note that no cosmological $K$-correction was made to the observed continuum fluxes. Given the small redshift of NGC $4051(z=0.0023)$, any $K$-correction is negligible and uncertainties in absolute flux calibrations are much larger than this correction. Table 1 gives the wavelength range and observation dates as well as references to the published works we have used to construct the broadband SED.

\section{MODELING THE SED}

The continuum jet emission in the outflow-dominated model we have used here is based on previous works by Maitra et al. (2009a) and Markoff et al. (2005). We refer the reader to these works for the details of the model. The main parameters that determine the properties of the jet in this model are the input jet power $\left(N_{\mathrm{j}}\right)$, electron temperature of the relativistic thermal plasma entering at the jet base $\left(T_{\mathrm{e}}\right)$, the ratio of magnetic to particle energy density (a.k.a. the equipartition factor $k$ ), physical dimensions of the jet base (assumed to be cylindrical with radius $r_{0}$ and height $h_{0}$ ), and the location of the point on the jet $\left(z_{\text {acc }}\right)$ beyond which a fraction of the leptons are accelerated to a power-law energy distribution $\left(d N / d E \sim E^{-p}\right) . N_{\mathrm{j}}$, parameterized in terms of the Eddington luminosity, determines the power initially input into the particles and magnetic field at the base of the jets. Beyond the jet base, we assume that the jet plasma (1) accelerates mildly due to a longitudinal pressure gradient with its bulk Lorentz factor asymptotically reaching a value of 2-3 far downstream along the jet, (2) expands laterally with its initial sound speed, and (3) cools due to adiabatic expansion. Once the initial conditions are specified, then the bulk speed of the jet plasma, magnetic field, and particle density are computed by solving the adiabatic, relativistic Euler equation (Falcke 1996). We note that in AGN, a combination of higher powers, confinement and perhaps stronger magnetic field pressures may give higher bulk Lorentz factors.

The accretion disk is modeled as a multi-color blackbody with radial temperature profile $T \propto R^{-0.75}$ (Shakura \& Sunyaev 1973; Mitsuda et al. 1984), and is parameterized by $T_{\text {in }}$ and $r_{\text {in }}$, the temperature and radius at the inner edge of the disk. The thermal photons from the accretion disk are also included in the photon 
field of the jet for Compton scattering, although due to Doppler redshifting in the jet frame, this external Compton emission is usually much smaller than the synchrotron self-Compton (SSC).

Given the various uncertainties involved, e.g., in our understanding of the cause of the large X-ray variability, nonsimultaneity of data from different wavelengths (except for the VLA/EVLA data and the Chandra data, which were obtained simultaneously), contribution to the optical/UV flux from nonAGN components, dust heating, etc., a detailed statistical comparison between the data and the model would yield errors which will significantly underestimate the systematic uncertainties. In other words, the uncertainties involved are much larger than simply the deviation of model prediction from the data. Therefore, the best-match model here is " $\chi$-by-eye" and is shown by the line labeled "Total" in Figure 1. In Figure 2, we show the model variability when one of the parameters was varied by $\pm 10 \%$ and $\pm 20 \%$ from the best-match model while the remaining parameter values were fixed to those of the best-match model.

We adopted a distance of $10 \mathrm{Mpc}$ (Sani et al. 2010) and the recently revised reverberation mass estimate of $1.7 \times 10^{6} M_{\odot}$ (Denney et al. 2009) for NGC 4051. The outer radius of the accretion disk is not constrained by the data and was arbitrarily set to a value of $10^{8} r_{\mathrm{g}}$. The remaining parameters were obtained from the best match to the data.

Assuming that the radio originates in an optically thick, stratified jet, a comparison of the radio and Spitzer/IRS fluxes immediately shows that the Spitzer fluxes are at least an order of magnitude higher than the expected jet flux. The large slit width of the IRS (3".6) is primarily responsible for this. The high spatial resolution Gemini/NIFS spectrum shows that the AGN contribution is much less.

The soft X-ray continuum, obtained with Chandra simultaneously with the $8.4 \mathrm{GHz}$ VLA/EVLA observations (King et al. 2011), shows much more variability both in the power-law index and normalization. Such variations could arise due to rapid changes in the particle energization/acceleration/cooling processes occurring in small "knots" in the post-shock region of the jet, e.g., as seen in the blazar BL Lacertae objects by Marscher et al. (2008). A full understanding of particle acceleration in such jets is still lacking; here we use a particle energy distribution index $p=2.7$ which matches the observed $>10^{16} \mathrm{~Hz}$ SED quite well if the observed EUV and X-ray emission is largely due to optically thin synchrotron. The jet inclination was taken to be $50^{\circ}$. While not strongly constrained by the data, a much lower value $\left(\lesssim 40^{\circ}\right)$ of the inclination would cause a larger fraction of the Doppler-boosted synchrotron photons from the outer parts of the jet to overestimate the radio flux. Inclinations greater than $\sim 60^{\circ}$ are similarly not preferred. We note that this inclination is the same as the results by Christopoulou et al. (1997), who modeled the narrow-line region of NGC 4051 as an outflowing biconical region of inclination $50^{\circ}$, thus suggesting that the relativistic jet as well as the much slower outflowing narrow-line region are co-aligned. Since we assume a symmetric model where the jets are perpendicular to the accretion disk, this means that the inclination to the disk normal is also $50^{\circ}$.

Since the radio data do not show any hint of low-energy cutoff, the minimum length of the jet was set to $0.3 \mathrm{pc}$, which is the upper limit for the source radius of the central component detected by Giroletti \& Panessa (2009) using VLBI. Also, while searching for the best-match model more weight was given to the VLA A configuration data point of King et al. (2011) since that observation had the highest spatial resolution as well as the smallest error bar of all the radio observations presented in that paper.

The equipartition factor $(k)$ is not strongly constrained by the data, although we could not obtain good matches either for $k<0.5$ or for $k>10$, pointing toward a near-equipartition model. We therefore set $k=1$. In addition to the jet, an accretion disk with $r_{\text {in }}=130 r_{\mathrm{g}}$ and $T_{\text {in }}=2.8 \times 10^{4} \mathrm{~K}$ is required to model the SED. This however assumes that the entire optical and UV emission is from the accretion disk. As discussed in Section 4 (and perhaps as evidenced from the PAH lines in the Spitzer/IRS spectrum), some of the UV emission could be from an actively star-forming nuclear star cluster. Some of the optical/ $\mathrm{UV}$ radiation could also be due to reprocessing of the X-rays from the jet base by the outer accretion disk, as suggested by the time lags reported in Breedt et al. (2010). Therefore, the inner disk radius obtained here is more realistically an upper limit on the actual radius.

The pre-shock synchrotron is the emission from the base/launching region of the jet. While the data do not constrain it directly in this case (see, e.g., the solid orange curve in Figure 1), the physical conditions at the base determine the nature of the flow farther downstream along the jet in the model. In order to produce the observed radio emission (which arises in the optically thick, post-shock synchrotron regime) and the $\mathrm{X}$-rays (which in our model are originating mainly in a region close to the base where a fraction of the thermal leptons are accelerated to a nonthermal power-law distribution), only certain physical conditions and geometries of the base are allowed, and these choices $\left(r_{0}=4 r_{\mathrm{g}}, h_{0} / r_{0}=1, T_{\mathrm{e}}=4 \times 10^{10} \mathrm{~K}\right.$, $z_{\text {acc }}=5 r_{\mathrm{g}}$ ) lead to the observed pre-shock synchrotron emission. An input jet power of $1.7 \times 10^{-2} L_{\text {Edd }}$, initially input into the particles and magnetic field at the base of the jets, is needed to match the broadband SED. The inverse Compton (IC) component (solid blue line in Figure 1) is also not strongly required by the data; however, it is computed self-consistently by the model and we include it in the plot to show that the X-ray emission is indeed dominated by synchrotron radiation and not by inverse Comptonization. We also tried models where the high energy is primarily dominated by IC scattering. However, within the framework of jet models, an IC-dominated model requires very extreme and rather unphysical parameters (e.g., $r_{0}<r_{\mathrm{g}}$ and $h_{0} / r_{0}>20$ ) for the X-rays, and is still unable to model the $E U V E$ flux. Therefore, we favor a synchrotron origin for the $E U V E$ and soft X-ray flux.

\section{DISCUSSION AND CONCLUSIONS}

It has long been known that the so-called radio-quiet Seyfert galaxies are not entirely radio silent (see, e.g., Ulvestad \& Wilson 1989; Wilson 1991; and also Ho \& Peng 2001 for a discussion). Recent VLBI observations of some of the faintest radio cores in Seyfert galaxies have suggested the presence of jets (Giroletti \& Panessa 2009). In this work we have studied the broadband nuclear emission from NGC 4051, a narrow line Seyfert galaxy, where the presence of a jet is suggested not only from radio VLBI, but also from observations at other wavelengths, e.g., the presence of shocked $\mathrm{Br} \gamma$ emission seen in the high-resolution Gemini/NIFS spectrum of the nuclear region (Riffel et al. 2008).

The modeling presented in this work suggests that once contributions from the host galaxy are properly accounted for, the broadband nuclear SED of the narrow line Seyfert galaxy NGC 4051 can be reasonably well described by emission 

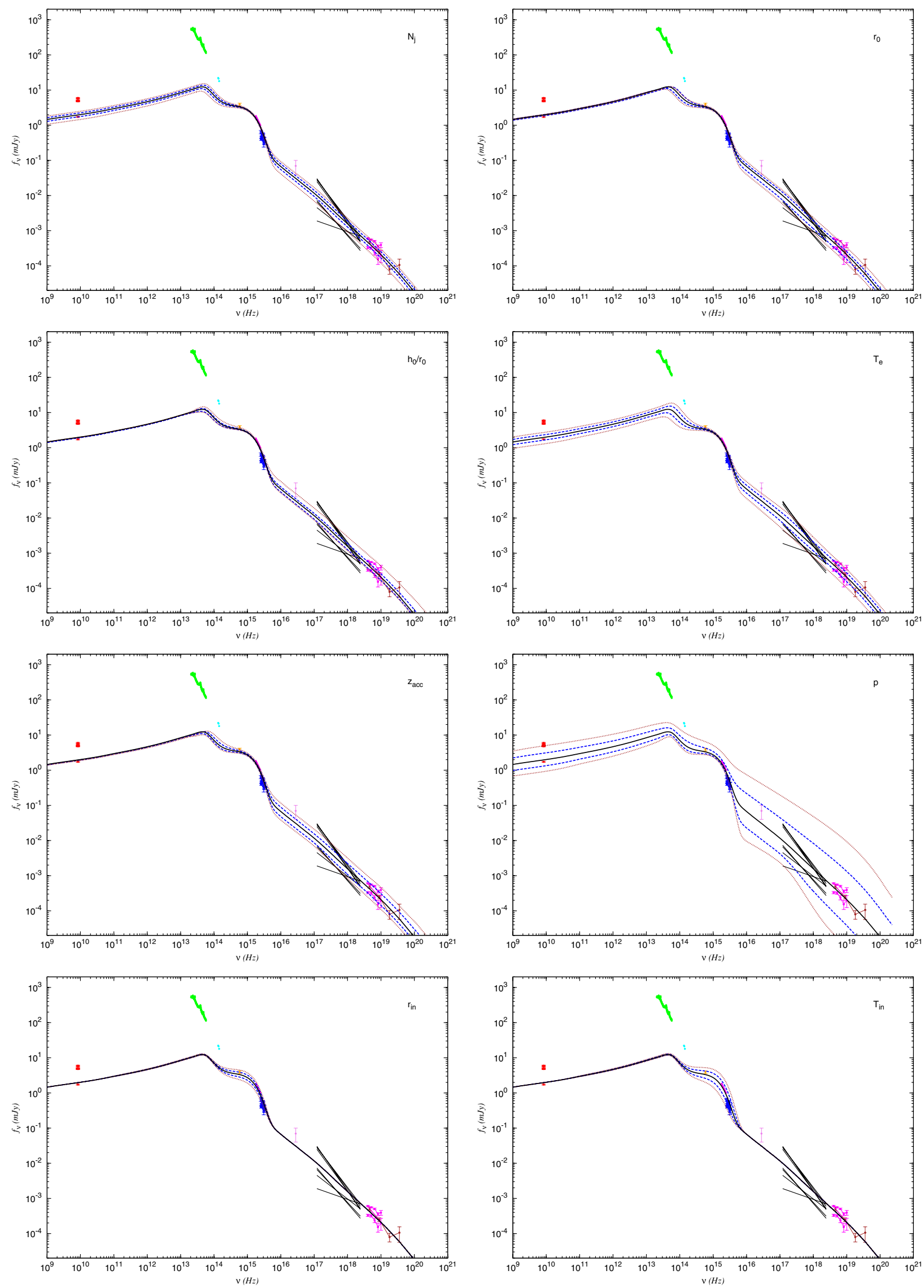

Figure 2. Solid black line in each of the above panels is the best-match model SED shown in Figure 1. The dashed blue lines show the SED when one of the jet model parameters (labeled near the top-right for each panel) was varied by $\pm 10 \%$. The remaining parameters were fixed to the best-match model. The dotted brown lines show the corresponding variability in the SEDs when the model parameter is changed by $\pm 20 \%$.

(A color version of this figure is available in the online journal.) 
Table 2

Comparison of Jet Model Parameters for Different Sources

\begin{tabular}{lcccc}
\hline \hline Parameter (unit) & NGC 4051 & HS-XRBs & Sgr A* & M81* \\
\hline$N_{\mathrm{j}}\left(L_{\text {Edd }}\right)$ & $1.7 \times 10^{-2}$ & $10^{-7}-10^{-1}$ & $10^{-8}$ & $10^{-5}$ \\
$r_{0}\left(r_{\mathrm{g}}\right)$ & 4 & $2-100$ & 2.5 & 2.4 \\
$h_{0} / r_{0}$ & 1 & $0.4-1.7$ & $0.4-2.5$ & $5-15$ \\
$T_{\mathrm{e}}(\mathrm{K})$ & $4 \times 10^{10}$ & $2-5 \times 10^{10}$ & $10^{11}$ & $10^{11}$ \\
$k$ & $1^{*}$ & $1-5$ & $>10$ & 1.4 \\
$z_{\text {acc }}\left(r_{\mathrm{g}}\right)$ & 5 & $10-400$ & $>10^{4}$ & 144 \\
$p$ & 2.7 & $2.4-2.9$ & $>3.8$ & 2.4 \\
\hline
\end{tabular}

Notes. The values for NGC 4051 are based on this work, and the others taken from a compilation of prior published literature by Markoff et al. (2011). An asterisk $(*)$ next to a parameter value indicates that the parameter was not varied while searching for the best-match model.

from an accretion disk plus a mildly relativistic jet outflow. A comparison of the best-match model parameter values obtained for NGC 4051 with XRBs in hard state and the LLAGNs Sgr A* and $\mathrm{M} 81 *$ is given in Table 2. The jet parameters for NGC 4051, viz. input power $\left(N_{\mathrm{j}}\right)$, geometry of the launching region $\left(r_{0}, h_{0}\right)$, the temperature of the thermal plasma $\left(T_{\mathrm{e}}\right)$, equipartition factor $(k)$, particle (lepton) energy distribution index $(p)$ are in the same ballpark regime for stellar mass black hole XRBs in hard state, even though the masses are different by five orders of magnitude. However, the location of the region beyond which particles are accelerated $\left(z_{\text {acc }}\right)$ appears to be closer to the central engine for NGC 4051 when compared to the LLAGNs. Broadband SED of more NLS1s would be needed to confirm whether NGC 4051 is an anomalous source in this respect, or if this is a generic feature of NLS1s. From our modeling it appears that the jet axis may be co-aligned with the outflowing narrow-line region (see, e.g., Christopoulou et al. 1997).

Even though there are not enough high-quality high energy $(>20 \mathrm{keV}$ ) data to constrain the efficiency of particle acceleration in the post-shock jet, the Swift/BAT data suggest that the high-energy cutoff, if present at all, is at $\gtrsim 100 \mathrm{keV}$. Similarly, it is not possible to constrain the equipartition factor strongly in this case, but models near equipartition or slightly magnetically dominated models are preferred. The location of the region where acceleration starts $\left(z_{\mathrm{acc}} \sim 5 r_{\mathrm{g}}\right.$ in this case) is still a free parameter in the model. Recent calculations by Polko et al. (2010) have indicated that it is possible to derive the location of this acceleration region self-consistently within the self-similar magnetohydrodynamical approximation proposed by Vlahakis \& Königl (2003). Once gravity is incorporated into this new calculation, we will be able to reduce the number of free parameters significantly in future versions of the jet model (P. Polko et al. 2011, in preparation).

\subsection{Variability}

The high variability of the X-rays and the extreme UV, and the fact that they are simultaneous to within $1 \mathrm{ks}$ (Uttley et al. 2000), suggests a common origin for these two bandpasses. The variability of the optical and lower frequencies is much smaller. In the framework of our model, the EUV and X-rays originate in a compact jet close to the central black hole. The observed high variability is expected both because of the small size of the emission region and relativistic beaming due to bulk motion of the jet plasma. The optical, on the other hand, originates from a much larger region in the accretion disk, and therefore exhibits much lower variability.
The energy distribution index of the shocked leptons ( $p=$ 2.7) obtained here is somewhat steeper than the values predicted, e.g., by diffusive shock process (Heavens \& Drury 1988). It is however possible that for NGC 4051 the EUVE and $\mathrm{X}$-ray bandpasses are at a higher energy than the cooling break energy, where the particle energy distribution steepens from $p$ to $p+1$ due to enhanced cooling. If the cooling break is indeed at a frequency lower than the $E U V E$, an immediate effect of this would be to move the optically thick to optically thin transition, currently in the $\sim 10-15 \mu \mathrm{m}$ range, to submillimeter wavelengths (where this break has been seen for the LLAGNS Sgr A* (Falcke \& Markoff 2000; Melia \& Falcke 2001; Maitra et al. 2009b) and M81* (Markoff et al. 2008)). Since the location of the cooling break is dependent on time as well as particle injection/acceleration rate, some of the observed variability could also be attributed to cooling.

Ponti et al. (2006) and Miller et al. (2010) have noted that above $\gtrsim 5 \mathrm{keV}$ there may be a tendency for the rms variability to decrease with increasing energy. X-ray flux in our model originates in the optically thin synchrotron from a distribution of shocked particles beyond $z_{\text {acc }}$. The spatial extent of such shocks may be small, and the X-ray variability could be due to rapid changes in particle energization, e.g., perhaps due to magnetic reconnection events. While the soft X-rays $(<10-20 \mathrm{keV})$ originate mainly due to this "post-shock" synchrotron, it is possible that IC scattering (of synchrotron photons produced at the base of the jet (SSC) as well as that of the soft photons from the accretion disk) becomes dominant at $>20 \mathrm{keV}$. Assuming a reasonably constant mass accretion rate by the disk, and a constant fraction of mass channeled into the jets, the variability of this IC component would be small and would largely reflect the (more slowly occurring) changes in mass accretion rate.

The simultaneous Chandra and VLA data presented by King et al. (2011) suggest that during their 2008-2009 campaign NGC 4051 shows an anti-correlation between X-rays and radio. Another recent work by Jones et al. (2011), using RXTE and VLA observations made during 2000-2001, found a weak positive correlation between radio and X-ray fluxes. The origin of the anti-correlation observed by King et al. (2011) is still not clear; however, this may be due to the following two reasons: (1) given the uncertainties in the bulk speed of the jet plasma, the simultaneous (as observed by us) radio and X-ray fluxes may not be causally connected, and (2) as multiwavelength monitoring results of Sgr A* show (see, e.g., Eckart et al. 2006; Yusef-Zadeh et al. 2008), there seems to be no tight correlation between the $\mathrm{X}$-ray and radio flares. In Sgr A* the optically thin NIR/X-ray flares most likely trace particle acceleration/(re)energization and cooling very close to the black hole, which only marginally affects the optically thick radio flux (Markoff et al. 2001). Changes in radio flux on the other hand are mainly driven by adiabatic expansion of overdensities in the outflow (Maitra et al. 2009b). It is also possible that NGC 4051 is in a transitional phase between hard and soft states, where the radio emission is in the process of getting quenched. While comparing with XRBs one must however keep in mind that the observed "quenching" of XRB jets may not mean that the jets have actually turned off. It is possible that XRBs jets may only appear quenched because they have become quite weak and the sensitivity is not enough to detect them. The presence of a separate mode for the disk-jet coupling (i.e., separate from the coupling which gives rise to the fundamental plane relation) operating in NGC 4051 during the King et al. (2011) observations cannot be ruled out either. 


\subsection{The Inner-edge Radius}

Our modeling suggests that the inner edge of the accretion disk is $\sim 130 r_{\mathrm{g}}$. Given that the model luminosity is $\sim 10^{-2}$ $L_{\text {Edd }}$, a large $r_{\text {in }}$ is not unreasonable. The profile of the iron $\mathrm{K} \alpha$ emission line near $6.4 \mathrm{keV}$ (see, e.g., Ponti et al. 2006; Miller et al. 2010), whose origin is generally attributed to hard $\mathrm{X}$-rays irradiating the relatively colder accretion disk (Reynolds \& Nowak 2003; Miller 2007), is quite narrow and does not show strong relativistic broadening, further suggesting that the accretion disk may be truncated at a large radius. However, by comparing the observed variability in the reflection features seen in the X-ray data with reflection models that include lightbending, Ponti et al. (2006) have suggested a much smaller inner-edge radius (within a few $r_{\mathrm{g}}$ ). We would like to point out that our estimation of the disk's $r_{\text {in }}$ assumes that the optical, UV, and FUV light are entirely coming from the accretion disk. However, ongoing star formation, as evidenced by the PAH lines in the Spitzer spectrum, may contribute some fraction (although most likely not a large fraction; see below) of the light that we are currently attributing to the disk. Any (presumably small) contribution from star formation will reduce the disk contribution, which in turn will likely imply a smaller disktruncation radius (assuming that $T_{\text {in }}$ of the accretion disk, which determines the peak of the optical/UV SED, is not changing).

An additional contributor to the optical and UV flux is the reprocessing of the more energetic EUV/X-rays from near the central black hole. A recent work studying the long-term optical and X-ray light curves of NGC 4051 by Breedt et al. (2010) hints at a lag of $1.2_{-0.3}^{+1.0}$ days, with the optical lagging behind the X-rays, thus favoring a reprocessing origin. On the other hand, results by Shemmer et al. (2003) suggest that part of the optical leads the X-ray by $\sim 2.4$ days. Based on their detailed reprocessing model, Breedt et al. (2010) concluded that there seems to be no common origin for the observed X-ray/optical variability and that not all of the observed optical variability can be attributed to reprocessing. Contribution by reprocessing would tend to lower the disk $r_{\text {in }}$. Therefore, the $r_{\text {in }}$ deduced here should perhaps more realistically be seen as an upper limit on the actual inner radius.

An independent measurement of the inner radius may come from a self-consistent modeling of the soft excess in this source. While the true nature of the soft excess is still debated (see, e.g., Gallo 2006; Middleton et al. 2007; Fabian et al. 2009), a survey of 34 type 1 AGNs by Crummy et al. (2006) and detailed spectral and timing analysis of the NLS1 galaxy 1 H0707-495 by Fabian et al. (2009) and Zoghbi et al. (2010) showed that the soft excess can be well modeled by a relativistically blurred photoionized disk reflection model. Assuming a maximally rotating Kerr black hole, disk reflection model fits to XMM-Newton data of NGC 4051 by Crummy et al. (2006) give an inner disk radius of $\sim 1.24 r_{\mathrm{g}}$. A more physical treatment of the scenario, where the $\mathrm{X}$-ray emission from the jet base (which can act as a source of irradiation) is self-consistently used to compute disk reflection features, would help in understanding soft excess seen in the source. If the inner disk radius is indeed close to the innermost stable circular orbit (ISCO) as suggested by the reflection model fits, this would require most of the flux, which is attributed to the accretion disk in this model, to have a non-disk origin.

\subsection{Stellar Contribution in the Optical/UV}

The HST/STIS spectrum given in Figure 6 of Collinge et al. (2001) suggests that the continuum flux between 1200 and
$1700 \AA$ is approximately constant at $F_{\lambda} \sim 2 \times 10^{-14} \mathrm{erg} \mathrm{cm}^{-2}$ $\mathrm{s}^{-1} \AA^{-1}$, implying an observed isotropic luminosity $L_{\mathrm{obs}}=$ $1.2 \times 10^{41}(D / 10 \mathrm{Mpc})^{2} \mathrm{erg} \mathrm{s}^{-1}$. In order to estimate the contribution from stellar flux at these wavelengths we used the synthetic SEDs of simple stellar populations published by Maraston (2005). We used a model SED with Salpeter (1955) initial mass function (IMF), solar metallicity, and red horizontal branch morphology (HBM). Since we are mainly interested in the blue/UV photons, the main factor that affects the luminosity is age, and the influence of other factors (IMF, metallicity, HBM) is comparatively much smaller. For the chosen set of parameters, the Maraston (2005) models give SEDs as a function of the age of the stellar population. Given the shape of the STIS continuum, a relatively young age of $\sim 0.1 \mathrm{Gyr}$ is needed. Integrating the $0.1 \mathrm{Gyr}$ Maraston model between 1200 and $1700 \AA$ gives a model luminosity of $L_{\text {model }}=3.25 \times 10^{33} \mathrm{erg} \mathrm{s}^{-1}$. Therefore, $L_{\text {obs }} / L_{\text {model }} \sim 3.7 \times 10^{7}$. Since the Maraston models are normalized to $1 M_{\odot}$, this implies that $\sim 37$ million young stars would be needed within the STIS aperture in order to have the same luminosity. Such a stellar concentration, while not implausibly large, is still at the high end of what has been observed in globular clusters and in nuclear star clusters (see, e.g., Côté et al. 2006).

However, there is a very important issue here that we have not considered: in reality it is well known that the stars in globular clusters are quite old, and so are the stars in the bulge. Nuclear star clusters typically consist of a somewhat older stellar population. According to the Maraston (2005) models, the number of stars needed to produce the STIS observed luminosity would have to be increased by four orders of magnitude if the stellar population was $1 \mathrm{Gyr}$ old, instead of the assumed $0.1 \mathrm{Gyr}$. On the other hand, recent results from Spitzer spectroscopy by Sani et al. (2010) show strong PAH emission at $6.2 \mu \mathrm{m}$ from narrow line Seyferts, indicating ongoing star formation within the circumnuclear region included inside the IRS's slit width (3".6). Clearly, high-resolution MIR observations would be required to have a better understanding of ongoing star formation in the nuclear region of NLSs. The variability seen in the optical wavelengths however argues against a scenario where stellar emission dominates the total observed optical flux. Based on our modeling, $<10 \%$ of the Spitzer observed flux is due to the jet. The jet contribution is higher in the $0^{\prime \prime} 1 \mathrm{Gemini}$ spectrum, but still appears to be dominated by sources other than the accretion disk and the jet.

The results of modeling the broadband SED of NGC 4051, along with similar results from modeling stellar mass black holes as well as LLAGNs such as Sagittarius $\mathrm{A}^{*}$ and M81*, broadly suggest that the underlying physical mechanism driving accretion inflows and outflows does not depend on the mass of the accretor and rather most of the observed properties scale with mass. Evidence for the presence of this mass scaling is not only seen in broadband spectral modeling and the existence of the fundamental plane, but also in various other recent observations which include (but are not limited to) detection of quasi-periodic oscillations in the NLS1 galaxy RE J1034 + 396 (Gierliński et al. 2008; Maitra \& Miller 2010; Middleton \& Done 2010), variability (see McHardy 2010 for a recent review), frequencydependent lags between energy bands (Arévalo \& Uttley 2006, and references therein), and iron line and other associated disk reflection features (see, e.g., Reynolds \& Nowak 2003; Miller 2007). It is interesting to note that recent X-ray and optical observations of gravitationally lensed quasars RX J1131-1231 and HE 1104-1805 suggest that the X-ray-emitting regions of these 
quasars are quite compact ( $\sim 10 r_{\mathrm{g}}$ or smaller; see, e.g., Chartas et al. 2009; Dai et al. 2010). As our modeling of NGC 4051 presented here shows, such a compact region of emission is expected if the X-rays are produced at the base of a jet.

Typically the inner disk radius is close to that of the ISCO in XRBs in soft state, whereas the inner disk radius we obtain here is much larger and more characteristic of hard state XRBs where the disk is truncated (although the radius we obtain is most likely an upper limit as discussed above, but otherwise it would require a rather improbably high number of extremely young stars to produce the observed UV flux). Based on our modeling, the continuum luminosity of the accretion disk is $6.1 \times 10^{41} \mathrm{erg} \mathrm{s}^{-1}$ $\left(\sim 0.3 \% L_{\text {Edd }}\right)$ and the isotropic luminosity from the power-law component between $10^{16}$ and $10^{19.5} \mathrm{~Hz}$ is $1.33 \times 10^{42} \mathrm{erg} \mathrm{s}^{-1}$ $\left(0.6 \% L_{\text {Edd }}\right.$, about twice the disk flux $)$. Integrating the broadband model flux between $10^{9}$ and $10^{19.5} \mathrm{~Hz}$, and assuming isotropic emission, gives a luminosity of $2.3 \times 10^{42} \mathrm{erg} \mathrm{s}^{-1}$ or $\sim 1.1 \% L_{\text {Edd }}$, in good agreement with the bolometric luminosity obtained with the $L_{\text {bol }} \approx 9 \lambda L_{\lambda}(5100 \AA)$ relation. Therefore, while the timing properties of NGC 4051 are similar to soft state XRBs, the SED and luminosities are more akin to hard state XRBs. Thus, it is unclear if the XRB state definitions can be straightforwardly carried over to AGNs, or if perhaps NGC 4051 is in a transitional phase between hard and soft states.

It would be interesting to test whether outflow-dominated models can explain the SED of other Seyfert galaxies (D. Maitra et al. 2011, in preparation). As shown in this work, isolating the AGN emission from starburst, dust, etc., is one of the biggest challenges for this project; and it is only in the very nearby Seyferts that we currently have the required resolution and sensitivity to see these relatively weak jets. The enhanced sensitivity of the EVLA at radio frequencies, and ALMA in millimeter and submillimeter wavelengths will soon provide data with unprecedented sensitivity and coverage, zooming into the heart of the central engines of the nearby AGNs to study accretion/ejection processes in much greater detail than before.

We thank Luigi Gallo for providing the Spitzer spectrum of NGC 4051. D.M. thanks Arunav Kundu, Marta Volonteri, and Kayhan Gültekin for discussions about stellar population models and quasar luminosity functions. Additionally, D.M. thanks John Causland for giving him a visual glimpse of NGC 4051 through his $24^{\prime \prime}$ reflector. S.M. gratefully acknowledges support from a Netherlands Organization for Scientific Research (NWO) Vidi Fellowship and from the European Community's Seventh Framework Programme (FP7/2007-2013) under grant agreement number ITN 215212 “Black Hole Universe.” J.M.M. is grateful for support through the Chandra Guest Observer program. We thank an anonymous referee for suggestions that led to improvements in the paper.

\section{REFERENCES}

Abdo, A. A., et al. 2010, ApJS, 188, 405

Arévalo, P., \& Uttley, P. 2006, MNRAS, 367, 801

Bentz, M. C., Peterson, B. M., Netzer, H., Pogge, R. W., \& Vestergaard, M. 2009, ApJ, 697, 160

Boller, T., Brandt, W. N., \& Fink, H. 1996, A\&A, 305, 53

Breedt, E., et al. 2010, MNRAS, 403, 605

Cardelli, J. A., Clayton, G. C., \& Mathis, J. S. 1989, ApJ, 345, 245

Chartas, G., Kochanek, C. S., Dai, X., Poindexter, S., \& Garmire, G. 2009, ApJ, 693, 174

Christopoulou, P. E., Holloway, A. J., Steffen, W., Mundell, C. G., Thean, A. H. C., Goudis, C. D., Meaburn, J., \& Pedlar, A. 1997, MNRAS, 284, 385

Collinge, M. J., et al. 2001, ApJ, 557, 2
Côté, P., et al. 2006, ApJS, 165, 57

Crummy, J., Fabian, A. C., Gallo, L., \& Ross, R. R. 2006, MNRAS, 365, 1067

Dai, X., Kochanek, C. S., Chartas, G., Kozłowski, S., Morgan, C. W., Garmire, G., \& Agol, E. 2010, ApJ, 709, 278

Denney, K. D., et al. 2009, ApJ, 702, 1353

Done, C., Ward, M. J., Fabian, A. C., Kunieda, H., Tsuruta, S., Lawrence, A., Smith, M. G., \& Wamsteker, W. 1990, MNRAS, 243, 713

Eckart, A., et al. 2006, A\&A, 450, 535

Elvis, M., Wilkes, B. J., \& Lockman, F. J. 1989, AJ, 97, 777

Fabian, A. C., et al. 2009, Nature, 459, 540

Falcke, H. 1996, ApJ, 464, L67

Falcke, H., Körding, E., \& Markoff, S. 2004, A\&A, 414, 895

Falcke, H., \& Markoff, S. 2000, A\&A, 362, 113

Fender, R. 2006, in Cambridge Astrophys. Ser. 39, Compact Stellar X-ray Sources, ed. W. Lewin \& M. van der Klis (Cambridge: Cambridge Univ. Press), 381

Fender, R. P., Gallo, E., \& Jonker, P. G. 2003, MNRAS, 343, L99

Gallo, L. C. 2006, MNRAS, 368, 479

Gierliński, M., Middleton, M., Ward, M., \& Done, C. 2008, Nature, 455, 369

Giroletti, M., \& Panessa, F. 2009, ApJ, 706, L260

Gültekin, K., Cackett, E. M., Miller, J. M., Di Matteo, T., Markoff, S., \& Richstone, D. O. 2009, ApJ, 706, 404

Heavens, A. F., \& Drury, L. O. 1988, MNRAS, 235, 997

Ho, L. C. 2002, ApJ, 564, 120

Ho, L. C., \& Peng, C. Y. 2001, ApJ, 555, 650

Homan, J., \& Belloni, T. 2005, Ap\&SS, 300, 107

Hunt, L. K., Mannucci, F., Salvati, M., \& Stanga, R. M. 1992, A\&A, 257, 434

Jones, S., McHardy, I., Moss, D., Seymour, N., Breedt, E., Uttley, P., Kording, E., \& Tudose, V. 2011, MNRAS, 412, 2641

Kaspi, S., Brandt, W. N., Collinge, M. J., Elvis, M., \& Reynolds, C. S. 2004, AJ, 127,2631

Kaspi, S., Smith, P. S., Netzer, H., Maoz, D., Jannuzi, B. T., \& Giveon, U. 2000, ApJ, 533, 631

King, A., et al. 2011, ApJ, 729, 19

Maitra, D., Markoff, S., Brocksopp, C., Noble, M., Nowak, M., \& Wilms, J. 2009a, MNRAS, 398, 1638

Maitra, D., Markoff, S., \& Falcke, H. 2009b, A\&A, 508, L13

Maitra, D., \& Miller, J. M. 2010, ApJ, 718, 551

Maraston, C. 2005, MNRAS, 362, 799

Markoff, S. 2010, The Jet Paradigm (Lecture Notes in Physics, Vol. 794; Berlin: Springer), 143

Markoff, S., Falcke, H., Yuan, F., \& Biermann, P. L. 2001, A\&A, 379, L13

Markoff, S., Nowak, M., Maitra, D., Wilms, J., Gallo, E., Hynes, R., \& Plotkin, R. 2011, in Proc. IAU Symp. 275, Jets at all Scales, ed. G. Romero, R. Sunyaev, \& T. Belloni (Cambridge: Cambridge Univ. Press), 250

Markoff, S., Nowak, M. A., \& Wilms, J. 2005, ApJ, 635, 1203

Markoff, S., et al. 2008, ApJ, 681, 905

Marscher, A. P., et al. 2008, Nature, 452, 966

McHardy, I. 2010, The Jet Paradigm (Lecture Notes in Physics, Vol. 794; Berlin: Springer), 203

McHardy, I. M., Papadakis, I. E., Uttley, P., Page, M. J., \& Mason, K. O. 2004, MNRAS, 348, 783

Melia, F., \& Falcke, H. 2001, ARA\&A, 39, 309

Merloni, A., Heinz, S., \& di Matteo, T. 2003, MNRAS, 345, 1057

Middleton, M., \& Done, C. 2010, MNRAS, 403, 9

Middleton, M., Done, C., \& Gierliński, M. 2007, MNRAS, 381, 1426

Miller, J. M. 2007, ARA\&A, 45, 441

Miller, L., Turner, T. J., Reeves, J. N., Lobban, A., Kraemer, S. B., \& Crenshaw, D. M. 2010, MNRAS, 403, 196

Mitsuda, K., et al. 1984, PASJ, 36, 74

Nowak, M. A., Wilms, J., Heinz, S., Pooley, G., Pottschmidt, K., \& Corbel, S. 2005, ApJ, 626, 1006

Osterbrock, D. E., \& Pogge, R. W. 1985, ApJ, 297, 166

Peterson, B. M., et al. 2004, ApJ, 613, 682

Polko, P., Meier, D. L., \& Markoff, S. 2010, ApJ, 723, 1343

Ponti, G., Miniutti, G., Cappi, M., Maraschi, L., Fabian, A. C., \& Iwasawa, K. 2006, MNRAS, 368, 903

Rees, M. J. 1984, ARA\&A, 22, 471

Remillard, R. A., \& McClintock, J. E. 2006, ARA\&A, 44, 49

Reynolds, C. S., \& Nowak, M. A. 2003, Phys. Rep., 377, 389

Riffel, R. A., Storchi-Bergmann, T., Winge, C., McGregor, P. J., Beck, T., \& Schmitt, H. 2008, MNRAS, 385, 1129

Russell, D. M., Maitra, D., Dunn, R. J. H., \& Markoff, S. 2010, MNRAS, 405, 1759

Salpeter, E. E. 1955, ApJ, 121, 161

Sani, E., Lutz, D., Risaliti, G., Netzer, H., Gallo, L. C., Trakhtenbrot, B., Sturm, E., \& Boller, T. 2010, MNRAS, 403, 1246 
Seyfert, C. K. 1943, ApJ, 97, 28

Shakura, N. I., \& Sunyaev, R. A. 1973, A\&A, 24, 337

Shemmer, O., Uttley, P., Netzer, H., \& McHardy, I. M. 2003, MNRAS, 343, 1341

Sikora, M., Stawarz, Ł., \& Lasota, J.-P. 2007, ApJ, 658, 815

Ulvestad, J. S., \& Wilson, A. S. 1989, ApJ, 343, 659

Uttley, P., McHardy, I. M., Papadakis, I. E., Cagnoni, I., \& Fruscione, A. 2000, MNRAS, 312, 880

Vaughan, S., Uttley, P., Pounds, K. A., Nandra, K., \& Strohmayer, T. E. 2011, MNRAS, 413, 2489
Vlahakis, N., \& Königl, A. 2003, ApJ, 596, 1080

Wilms, J., Nowak, M. A., Pottschmidt, K., Pooley, G. G., \& Fritz, S. 2006, A\&A, 447,245

Wilson, A. S. 1991, in ASP Conf. Ser. 18, The Interpretation of Modern Synthesis Observations of Spiral Galaxies, ed. N. Duric \& P. C. Crane (San Francisco, CA: ASP), 227

Yusef-Zadeh, F., Wardle, M., Heinke, C., Dowell, C. D., Roberts, D., Baganoff, F. K., \& Cotton, W. 2008, ApJ, 682, 361

Zoghbi, A., Fabian, A. C., Uttley, P., Miniutti, G., Gallo, L. C., Reynolds, C. S., Miller, J. M., \& Ponti, G. 2010, MNRAS, 401, 2419 